\title{
Between the devil and the deep blue sea
}

Robert W. Cahn, Dean of the School of Applied Sciences at the University of Sussex, offers this comment on the prospect for fusion power

IN the anguished debates on the future, if any, of nuclear fission power, various recognisable strands emerge among the opposition. Some pin their faith on the improved technological exploitation of familiar fossil sources, principally coal; others would drive us headlong into the solar economy, often conceived in terms of 'small is beautiful' and a rural life of log-cabin simplicity; yet others accept the need for high technology on a very large scale if the sun is to yield domestic warmth, electricity and liquid fuel on an adequate scale.

All three groups, however, have one characteristic in common. When faced by hard statistical or technological criticisms, they declare that we need only keep going for a few decades and then the boundless energy "from seawater"-that is, nuclear fusion-will become a reality and all our worries will be over. Although they do clearly recognise that the method is not altogether simple, even the most resolute proclaimers of radioactive doom seem to have convinced themselves that fusion power will be gentle and on a homely scale, and that radioactive pollution will be a thing of the past when dirty fission gives way to clean fusion. Probably the fact that fusion takes place in the sun and that the sun is the emblem of cleanliness and of life itself, has something to do with this touching faith. We are all to some degree subject to the ad-man's technique of fallacious association.

There is no dearth of popularisations of this very difficult and complex topic, but they all concentrate on two aspects of fusion-how to confine the plasma, and how to heat it to ignition temperature. Even within these limits, they tend to concentrate on scientific principles and not on engineering realities, whereas the current critcism of nuclear fission concentrates on real and alleged engineering weaknesses. Nuclear fusion has progressed far enough now towards the design of a self-sustaining reactor to make it both possible and necessary to see the problems in realistic terms. The plasma confiners-which indeed have a ferociously difficult task-until recently have hogged all the attention, to the neglect of the problems of energy balance (achieving a positive net energy output), container strength and fatigue damage, lithium supply, reactor cooling, injection of gas into the plasma without quenching it, and especially the radiation damage inside the reactor itself.

That this imbalance is now being repaired is primarily due to the efforts of the Energy Research and Develop- ment Administration (ERDA), which has complete control of fusion research in the United States. ERDA has prepared a detailed R\&D plan extending to 1990 , and in outline to the end of the century. ERDA's achievement is to have set out this programme without committing itself to any particular design of reactor, and yet to have identified the central problems which will have to be faced irrespective of the design. To do this, ERDA's predecessor in 1967 invented the concept of the 'reference design'. This is an outline based on scientific principles, as presently understood, set out in such a way as to identify, as quantitatively as possible, the whole range of engineering problems that will need to be mastered. Such a reference design is particularly illuminating when applied to the doughnut-shaped Tokamak, which in purely scientific terms is today probably the most hopeful contender among the rival approaches.

On the basis of such a reference design, the problem of securing the deuterium (by electrolysis of water?) and tritium from a metre-thick breeding blanket of molten lithium surrounding the first wall of a doughnut-shaped plasma device, can be analysed, not least in terms of lithium availability; lithium is a scarce element. One day it will also be necessary to include in the energy balance equation not only the energy cost of extracting tritium from irradiated lithium but also the electricity costs of electrolysing water to extract deuterium, a calculation which never seems to be reported.

Probably the most alarming engineering problem is that of radiation damage. In the much-criticised fission reactor, neutrons, gamma rays and fission particles remain inside the reactor volume, and indeed the fission products remain inside the nuclear fuel itself. In a Tokamak, all the neutrons, moving with a kinetic energy of some $14 \mathrm{MeV}$, beat upon the first wall: the scalc of this bombardment dwarfs anything in a fission reactor. The wall can be sputtered away, especially by the novel hazard of 'chunky sputtering' which involves the physical removal of thousands of atoms at a time.

A great deal of research is now in progress on this group of problems in the United States (some also in Britain), and considerable advances have been made in improving the radiation resistance of high-strength refractory alloys. But it is certainly an error to regard fusion reactors as generating little radioactivity: there will be a great deal of induced radioactivity, though not from fission products, and it will be just as crucial as in the case of a fission reactor to contain and seal off this radioactivity from the outer world. (It seems likely that this hazard will not be long-lived). Apart from this, there will be just the same hazards of pinholes developing in inaccessible locations as have been found with prototype fissionbreeder reactors, leading to deterioration in cooling efficiency or vacuum quality.

Fusion may prove to be a practical source of energy - the new issue is now as much in the hands of the engineers and metallurgists as of the plasma physicists. If it comes, it will be an immeasurable boon but it will also bring with it problems of pollution, energy imbalance during construction, resource depletion, sabotage and potential stoppage such as we find with every other source of energy, including solar techniques such as the large-scale fermentation of timber to make liquid fuel.

For Blake, energy was a mark of the divine. Milton knew better: Satan has energy too. Whether the external flames are fed by coal, oil, uranium or deuterium is a question which must tax the physical theologians of the future. 